\title{
THE EFFECTS OF STIMULUS INTENSITY AND AGE ON VISUAL-EVOKED POTENTIALS (VEPS) IN NORMAL CHILDREN.
}

\author{
Authors: María Teresa Carrillo de la Peña, Socorro Rodriguez Holguín, Fernando \\ Cadaveira
}

This is the peer reviewed version of the following article: Carrillo-de-la-Peña, M.T.; Rodríguez Holguín, S.; Cadaveira, F. (1999). The effects of stimulus intensity and age on visual-evoked potentials (VEPs) in normal children. Psychophysiology, 36(6), 693-698. https://doi.org/10.1111/1469-8986.3660693.

This article may be used for non-commercial purposes in accordance with Wiley and the Society for Psychophysiological Research terms and conditions for use of self-archived versions. 


\title{
The effects of stimulus intensity and age on visual-evoked potentials (VEPs) in normal children
}

\author{
M.T. CARRILLO-DE-LA-PEÑA¹, S. RODRÍGUEZ HOLGUÍN, M. CORRAL, F. CADAVEIRA \\ Department of Clinical Psychology and Psychobiology, University of Santiago de Compostela, Galicia, Spain
}

\begin{abstract}
In this study, we explored the effects of flash intensity and age on visual-evoked potentials (VEPs) in a sample of 85 children aged 8-15 years. Results of previous studies are discrepant regarding the extent to which children show an evoked potential augmenting tendency at vertex, which has been reported to be a characteristic of an immature inhibitory control system. In the present study, VEPs to light flashes of four different intensities were recorded at Cz. The results confirmed that $\mathrm{P} 1 \mathrm{~N} 1$ and N1P2 at Cz were positively related to increases in stimulus intensity, whereas $\mathrm{N} 1$ was not related reliably to intensity. This difference between peak-peak and baseline-peak amplitude findings at $\mathrm{Cz}$ relative to evoked potential augmenting and reducing may help to explain discrepant results among earlier studies. Developmental changes were found for our sample of children that were independent of stimulus intensity: N1 amplitude increased significantly with age, whereas N1 latency showed a small (nonsignificant) age-related decrease.
\end{abstract}

Descriptors: Visual-evoked potentials, Children, Intensity, Age, Augmenting/reducing (A/R)

The relationship between stimulus intensity and the magnitude of early evoked potential (EP) components is not straightforward. Although it has been reported that the N1 EP component increases in amplitude and decreases in latency with increasing stimulus intensity, the amplitude characteristics of this component vary widely across subjects when high intensities are used (Näätanen \& Picton, 1987). These individual differences in the modulation of sensory input have been the basis for research into cortical augmenting and reducing ( A/R). Following Petrie's original formulation of perceptual A/R (Petrie, 1960, 1978), Buchsbaum and Silverman (1968) evaluated EPs to four different intensities of light flashes and were able to differentiate individuals who showed increases in EP amplitude at higher intensities (augmenters) from other subjects whose EP amplitudes leveled off or decreased (reducers). Slope of EP peak-to-peak amplitude versus log intensity plots was used to measure A/R. According to normative data reported by Buchsbaum, Haier, and Johnson (1983), nearly $80 \%$ of the subjects had EPs whose amplitudes continued to increase in parallel with stronger intensities. When stricter criteria were adopted that required slopes larger than 1.0 for augmentation and zero or negative slopes for reduction, only $33.4 \%$ of their subjects were classified as augmenters and $19.6 \%$ as reducers. Buchsbaum and Pfefferbaum (1971) found that the A/R phenomenon was most evident in EPs recorded at vertex and that $\mathrm{P} 1 \mathrm{~N} 1$ was more affected than N1P2. They suggested that some kind of central inhibition mechanism was involved. Later studies supported the explanation of $A / R$ as a nonspecific phenomenon that is independent of sensory modality (Barratt, Pritchard, Faulk, \& Brandt, 1987; Orlebeke, Kok, \& Zeillemaker, 1984).

\footnotetext{
1 Address reprint requests to: María Carrillo-de-la-Peña, Departamento de Psicoloxía Clínica e Psicobioloxía, Facultade de Psicoloxía, Campus Universitario Sur, 15706, Santiago de Compostela, Galicia, Spain. E-mail: pcpbmtc@uscmail.usc.es.

This research was supported by the Spanish Ministry of Education and Culture (DGICYT) grant PB95-0856.
}

Received March 16, 1998; Accepted November 12, 1998 
Relatively few studies have investigated the effects of stimulus intensity on EPs of normal children. For the visual modality, age-related differences in cortical responsiveness to flashes of different intensities have been reported. Dustman and Snyder (1981) found that bright flashes resulted in larger amplitude increases in visual-evoked potentials (VEPs) from frontocentral areas of children and old persons when compared with adults of an intermediate age. They interpreted their finding as being an index of a protective frontocentral inhibitory mechanism that was diminished in the youngest and oldest subjects. This result was confirmed in later studies that also demonstrated that amplitude0intensity slopes of VEPs from frontocentral areas of children were larger than those of young adults (Dustman, Shearer, \& Emmerson, 1993; Dustman, Snyder, \& Schlehuber, 1981). However, results from a study using auditory stimuli (Bruneau, Roux, Guérin, Barthélémy, \& Lelord, 1997) did not show an augmenting response at vertex scalp $(\mathrm{Cz})$ in 4-8-year-old children; the modulatory effects of intensity on N1 amplitude were only prominent at temporal sites. Apart from the different stimulus modality, they considered different EP components. Dustman and co-workers analyzed the effects of stimulus intensity on peak-to-peak amplitudes or on amplitude-intensity slopes, which were computed on a composite measure that included the joint amplitudes of three VEP components occurring between 90 and $200 \mathrm{~ms}$ following stimulus onset, whereas Bruneau et al. (1997) analyzed only peak-to-baseline N1 amplitude.

The phenomenon of $A / R$ in children needs further investigation given its potential clinical utility for the evaluation of treatment efficacy as studies of hyperactive and autistic children have documented. Dykman, Holcomb, Ackerman, and McCray (1983) found that the ameliorative effects of methylphenidate were more evident in hyperactive children who demonstrated auditory EP (AEP) augmenting patterns for $\mathrm{N} 1-\mathrm{P} 2$, and it has been reported that autistic responders to treatment with fenfluramine tended to be augmenters in the auditory modality (Bruneau, Barthélémy, Roux, Jouve, \& Lelord, 1989).

The present study explored the extent to which a deficient inhibitory control system was present in children as indexed by a vertex VEP augmenting tendency of N1, and P1N1 and N1P2 amplitude values. VEPs at vertex were expected to be sensitive to changes in stimulus intensity. To elucidate whether the $A / R$ tendency is modified by age, two groups of children were studied: younger (8-11 years) and older (12-15 years). As there are few reports of VEP normative data for this age range, an additional objective of this study was to explore if the amplitude and latency of N1 in VEPs elicited by flashes of different intensities changed with age.

\section{Method}

A total of 85 children aged $8-15$ years $(M=11.4 \pm 2.24$ years) were assessed. Subjects were divided into two age groups: younger, that is, 8-11 years ( $n=42$, 21 females; $M=9.4 \pm 1.06)$ and older, that is, $12-15$ years ( $n=43,23$ females; $M=13.4 \pm 1.04$ years). All participants were healthy with no history of psychopathological or neurological disorders. Other exclusion criteria such as current medication, consumption of alcohol or drugs that might affect VEPs in some way either from current or past use, prenatal exposure to alcohol or other drugs, developmental or educational disability, and noncorrected sensory or motor deficits, were also considered. Twenty children contacted were excluded on the basis of these criteria.

Stimuli were light flashes generated by a Grass Model PS-22 Photostimulator (Grass Instruments, Quincy, MA), which was positioned $100 \mathrm{~cm}$ in front of the subject. In each EP recording session, four blocks of 75 flashes were presented; each block had stimuli of the same intensity. Grass Photostimulator settings of $1,4,8$, and 16 were used; these settings are equivalent to 93,750, 375,000, 750,000, and $1,500,000$ candles, respectively. Connolly and Gruzelier (1982) argued that it is best to measure flash intensity at the subject's position, but because the flashes were too short for intensity measurement by available light meters, we assumed that the intensities were those listed in the instruction manual for 
Model PS2 and PS3 Photostimulators. The interstimuli interval was $1 \mathrm{~s}$, and the inter-blocks interval was 2 min. The order of presentation of the four intensities was assigned randomly across subjects.

Subjects sat in a comfortable armchair in an electrically isolated, sound- and light-attenuated room. They were instructed to fix their eyes on a point in front of them, not to look directly at the flashes, and to avoid movements during the test.

For this report, electroencephalogram (EEG) activity was recorded with tin electrodes at $\mathrm{Cz}$ (standard electrode position nomenclature, American Electroencephalographic Society, 1991) and referred to linked earlobes. An electrode placed at forehead served as ground. Additional electrodes placed supraorbitally and infraorbitally of the left eye were used to monitor ocular artifacts. EEG was amplified $20 \mathrm{~K}$ and filtered using a bandpass of 1-100 Hz (Grass Neurodata Acquisition System, Mod. 12, connected to a NeuroScan Inc. system for the analog-to-digital conversion and storage). Impedances were kept at $5 \mathrm{~K} \Omega$ or less. EEG was sampled at a rate of $256 \mathrm{~Hz}$ with an epoch length of $500 \mathrm{~ms}(50 \mathrm{~ms}$ prestimulus baseline). Epochs with excessive eye or body movements $( \pm 100 \mu \mathrm{V})$ were rejected. Four averaged waveforms (one per intensity level) with at least 60 artifact-free epochs were obtained for each subject.

The averaged VEPs were analyzed with a semiautomatic peak detection program, which examined latency windows of $60-120,80-180$, and 110-280 ms for P1, N1, and P2 peaks, respectively. Peaks were then verified and adjusted following visual inspection. Amplitude and latency values were automatically exported to an ASCII file for subsequent analyses. Both baseline-to-peak amplitudes $(\mu \mathrm{V})$ and peak latencies (ms), as well as the peak-to-peak amplitudes $(\mu \mathrm{V})$ of P1-N1 and N1-P2, were measured for each subject at each intensity level.

Data were analyzed with a mixed-model analysis of variance (ANOVA) using intensity (four levels) as a within-subject factor and age group (two levels) as a between-subject factor. Separate analyses were made for N1 latency, and N1, P1N1, and N1P2 amplitudes. Significance levels were determined using degrees of freedom adjusted by the Greenhouse-Geisser correction when violation of the assumption of sphericity was found significant (Vasey \& Thayer, 1987). Preliminary analyses also included gender as a between-subject factor. However, as we did not obtain significant Gender, Gender x Intensity or Gender x Age effects, this factor was not included in subsequent analyses.

The slope of linear equation obtained by least squares regression of amplitude on the logarithm (base $10 \mathrm{log}$ ) of flash intensity (in candles) was used to assess the relative prevalence of "augmenter" and "reducer" children. Amplitude-intensity slope was calculated for the mean of the two peak-to-peak amplitudes, that is, (P1N1 + N1P2) / 2. This measure was used by Dustman et al. (1993).

\section{Results}

\section{Evaluation of EP Waveforms}

The grand averages of VEPs from $\mathrm{Cz}$ for each flash intensity and both age groups are presented in Figure 1. Visual inspection of the waves revealed that amplitudes were larger for the 12-15-year-old subjects for each intensity. Also, VEP amplitudes for both age groups generally increased in size as intensity was increased.

\section{N1 Latency and Amplitude}

The mean N1 latency and amplitude values at the different intensity levels for each group of age are presented in Table 1. Repeated-measures ANOVA showed that the within-subject factor "intensity" was only significant for $\mathrm{N} 1$ latency, $F(3,243)=4.67 ; p=.005 ; \varepsilon=0.89$. N1 latencies decreased with increasing 
intensity, whereas amplitude values were unaffected. The between-subject factor "age group" was significant for $\mathrm{N} 1$ amplitude values, $F(1,81)=6.27 ; p=.014$. The oldest group (12-15 years) showed significantly larger (more negative) N1 amplitudes. This effect was independent of stimulus intensity.
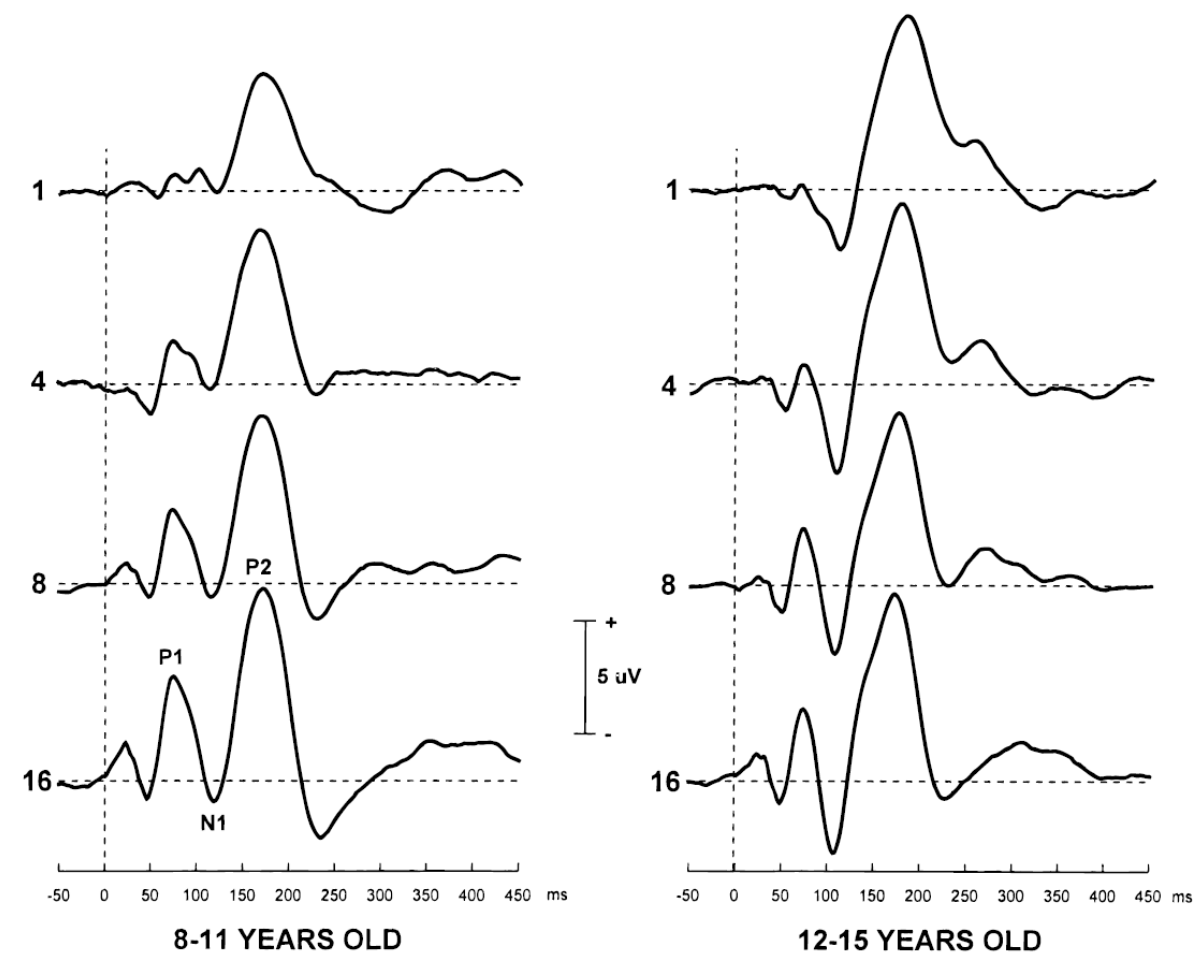

Figure 1. Grand averages of visual-evoked potentials (VEPs) that were recorded from two groups of subjects aged 8-11 and 12-15 years and elicited by four increasing intensities of light flashes $(1,4,8$, and 16).

Table 1. Mean (SD) latency and amplitude values for $N 1$ at the four different intensities for each age group

\begin{tabular}{lcccc}
\hline \hline \multicolumn{5}{c}{ Intensity } \\
\cline { 2 - 5 } Age group (years) & 1 & 4 & 8 & 16 \\
\hline N1 latency (ms) & & & & \\
$\quad 8-11$ & $117.69(18.61)$ & $112.73(17.42)$ & $113.39(16.77)$ & $114.55(15.82)$ \\
$\quad 12-15$ & $113.07(15.10)$ & $110.17(12.02)$ & $11.56(14.60)$ & $110.17(12.84)$ \\
N1 amplitude $(\mu \mathrm{V})$ & & & & \\
$8-11$ & $-2.44(3.73)$ & $-2.70(4.28)$ & $-2.85(4.59)$ & $-3.08(5.18)$ \\
$12-15$ & $-4.25(3.30)$ & $-5.25(4.12)$ & $-4.81(4.12)$ & $-4.95(4.66)$ \\
& & & & \\
\hline \hline
\end{tabular}

\section{Peak-to-Peak Amplitudes}

Repeated-measures ANOVAs were performed separately for P1N1 and N1P2 amplitudes. Table 2 shows mean amplitudes for each of the Intensity $x$ Age conditions. The effect of intensity was significant for P1N1 and N1P2 ( $p .001$; F-ratios $[3,237 d f]$ were 31.84 and 9.66, respectively). For both components, amplitudes were positively related to flash intensity. 
A significant effect of age was found only for N1P2 amplitude, $F(1,79)=4.00 ; p=.049$. Amplitudes of the older children were larger than those of the younger children. The Age $x$ Intensity interaction was not significant.

Table 2. Mean (SD) amplitude values for P1N1 and N1P2 at the four different intensities for each age group

\begin{tabular}{lcccc}
\hline \hline & \multicolumn{4}{c}{ Intensity } \\
\cline { 2 - 5 } Age group (years) & 1 & 4 & 8 & 16 \\
\hline P1N1 amplitude $(\mu \mathrm{V})$ & & & \\
$8-11$ & $4.98(3.06)$ & $6.38(4.55)$ & $7.67(4.58)$ & $9.00(5.95)$ \\
12-15 & $5.93(2.43)$ & $7.40(3.48)$ & $8.68(3.85)$ & $9.72(4.45)$ \\
N1P2 amplitude $(\mu \mathrm{V})$ & $10.27(5.43)$ & $11.48(5.43)$ & $12.36(5.40)$ & $13.82(6.99)$ \\
$8-11$ & $13.91(5.45)$ & $14.76(5.79)$ & $14.56(5.98)$ & $15.42(6.98)$ \\
$12-15$ & & & & \\
\hline \hline
\end{tabular}

\section{Amplitude-Intensity Slope}

Amplitude augmentation was defined as slopes greater than 1.0 and amplitude reduction as slopes less than 0.0 . According to this criteria, $61.2 \%(n=52)$ of the 85 children were classified as augmenters and $20 \%(n=17)$ were classified as reducers. Children classified as augmenter $(A)$ or reducer $(R)$ were distributed among both age groups as follows: younger group: $27 \mathrm{~A}, 7 \mathrm{R}$ and older group: $25 \mathrm{~A}, 10 \mathrm{R}$. To explore whether augmenters from the two groups of age differed in the steepness of the amplitude 0 intensity slope, mean slope values were calculated for each group. For the augmenters in the 8- to 11year-old group the mean value of the slope was $4.83(S D=3.27)$ and for those of the 12- to 15-year-old group the mean was $4.13(S D=2.05)$. A one-way ANOVA revealed that this difference was not statistically significant. ${ }^{1}$

${ }^{1}$ Following a reviewer's suggestion, analyses were repeated dividing the children into four age groups. The frequency distribution of augmenters and reducers, and the slope values for the augmenter in each group are depicted in the following table:

\begin{tabular}{lcccc}
\hline $\begin{array}{l}\text { Age group } \\
\text { (years) }\end{array}$ & $N$ (females) & Augmenter & Reducer & $\begin{array}{c}\text { Mean } \\
\text { slope }\end{array}$ \\
\hline $8-9$ & $21(11)$ & 14 & 3 & 5.47 \\
$10-11$ & $21(10)$ & 13 & 4 & 4.14 \\
$12-13$ & $24(12)$ & 14 & 6 & 4.08 \\
$14-15$ & $19(8)$ & 11 & 4 & 4.19 \\
\hline \hline
\end{tabular}

The repeated-measures ANOVA confirmed the absence of interaction between age and intensity:

\begin{tabular}{lcc}
\hline \hline Age group $\times$ Intensity & $F$ & $p$ \\
\hline N1 & 1.02 & .425 \\
P1N1 & 0.29 & .976 \\
N1P2 & 1.29 & .245 \\
\hline
\end{tabular}

Also, no significant differences in the proportion of augmenters and reducers, or in the mean slope values were found among the four age groups, $F(3,81)=0.91, p .439$.

\section{Discussion}

A modulatory effect of stimulus intensity on VEPs recorded at Cz was observed for P1N1 and N1P2. Also, most of the children (61.2\%) showed VEP-augmenting amplitude-intensity slopes. The frequency distribution of augmenting and reducing children found in the present study was significantly different, $\chi^{2}=$ 
12.69, p.005 from that reported by Buchsbaum et al. (1983) on a sample of adults, in which only $33.4 \%$ were augmenters. Taken together, these results seem to confirm that augmenting is the more frequent pattern of sensory modulation among children and lend support to the hypothesis of immaturity of inhibition mechanisms in children as proposed by Dustman et al. (1993).

The above conclusion contrasts clearly with Bruneau et al.'s (1997) failure to find increases in AEP N1 amplitude with increasing stimulus intensity at midline sites in a sample of children. Several reasons may be at the basis of this discrepancy, such as differences in stimulus modality, intensity range, or interstimulus interval (ISI). It is well known that VEPs and AEPs constitute responses with different morphology and generators, and that results from one modality cannot be generalized easily to the other. In fact, multimodal studies have failed to observe A/R correlation between the visual and auditory modalities (Blenner \& Yingling, 1993; Buchsbaum et al., 1983; Kaskey, Salzman, Klorman, \& Pass, 1980; Lolas, Collin, Camposano, Etcheberrigaray, \& Rees, 1987; Raine, Mitchell, \& Venables, 1981). Given the relevance of intensity range in the definition of $A / R$ (see Carrillo-de-la-Peña, 1992 for a review), it is possible that the discrepancy stems from the use of stimuli not comparable in intensity in both modalities. Additionally, the inhibitory mechanism may have a different time course in each stimulus modality (the auditory system operates much more slowly than the visual system) and thus the differences in A/R might be explained by the ISIs used. In fact, Como, Simons, and Zuckerman (1984) found that AEPs and VEPs slopes correlated for a long ISI (17 s) but not for a short ISI (2 s).

Nevertheless, the fact that Bruneau et al. (1997) found modulatory effects of intensity on N1 amplitude only at temporal, and not at frontocentral, placements in a sample of children is in clear contradiction with the last studies on AEPs A/R in adults (Hegerl, Gallinat, \& Mrowinski, 1994; Hegerl \& Juckel, 1993) and non-human primates (Pineda, Homes, \& Foote, 1991). In these studies, it was found that the waveform recorded at frontocentral placements in the $\mathrm{N} 1$ latency range shows a stronger intensity depen dence than that recorded at temporal leads

Taking into account the above considerations, it is possible to' offer an alternative explanation of the Bruneau et al.'s (1997) findings. The lack of an AEP-augmenting pattern at midline sites in a sample of children may be explained by the fact that they considered only $\mathrm{N} 1$ peak-to-baseline amplitude. In this study, similarly, visual N1 amplitude was not sensitive to stimulus intensity changes, whereas P1N1 and N1P2 showed a modulatory effect of stimulus intensity.

The inconsistency due to the use of different EP components has been reported in previous literature on A/R. Prescott, Connolly, and Gruzelier (1984) found no agreement between the subject classifications (augmenter or reducer) for AEP components P1, N1, P2, P1N1, and N1P2. Nevertheless, this result seems discordant with other studies in human adults and in animals (cats) that found that N1 was sufficiently sensitive for defining A/R (Bruneau, Roux, Garreau, \& Lelord, 1985; Connolly \& Gruzelier, 1982) and the most stable component (Saxton, Siegel, \& Lukas, 1987). This discrepancy may be explained by the unreliability of the AEP N1 component recorded from central scalp of children as reported in developmental studies (Martín, Barajas, Fernandez, \& Torres, 1988), and could represent a difference between children and adults with implications for $A / R$ definition.

N1 latency was sensitive to the manipulation of stimulus intensity: latencies were shortened with the brightest flashes. Dustman, Shearer, and Snyder (1982) also found an inverse relationship between flash intensity and VEP component latency.

No significant interaction of intensity with age was found: VEP augmentation was not stronger for the younger than older children. The Dustman et al. (1993) study showed a rapid drop in amplitude0 intensity slope from about age 6 , although subjects were predominantly augmenters until the age of 17 . In the present study, slope values were slightly larger for the youngest group, but the difference was not significant. The age range of the sample used here (8-15 years) may not be wide enough to permit the observation of age-related changes in $A / R$. The results obtained confirm that changes in slope values do 
not occur before 15 years of age and are in agreement with studies into the maturation of visual EPs, which concluded that cortical developmental changes were not complete until 17 years of age or later (Allison, Hume, Wood, \& Goff, 1984).

The effect of age was significant for N1 amplitude: the older children showed greater N1 peak amplitudes than the younger children, and this difference was independent of stimulus intensity. This developmental effect has been reported for AEPs (Martín et al., 1988), whereas studies in the visual modality did not find a significant effect of age on N1 amplitude (Allison et al., 1984; Courchesne, 1978) or found decreases in amplitude with age (Johnson, 1989). With regard to N1 latency, our 12-15-year-old group was characterized by slightly shorter latencies, although this trend did not reach significance, in contrast to findings from other studies (Allison et al., 1984; Johnson, 1989). These discrepancies between this and previous studies may be due to the narrower range of age in the present study, because in the above-quoted studies the decrease in amplitude and latency appeared with age bands that included subjects aged 20 years or more.

The present study demonstrated that cortical augmenting is the most frequent pattern of sensory modulation in children and suggested the existence of reduced inhibitory control at this age range. The use of vertex only in the recordings is a limitation of the present work and a future investigation on this field should include more electrodes. As Dustman, Emmerson, and Shearer (1996) emphasized, neurobiological mechanisms underlying augmenting and reducing appear to be related to monoaminergic activity. Several studies that analyzed the functioning of the dopaminergic and serotonergic neurotransmitter systems, which are predominantly inhibitory in nature, have supported the view that inhibitory strength underlies individual differences in EP augmenting0 reducing; decreased serotonine and dopamine levels are associated with higher stimulus0amplitude slopes (Bruneau, Barthélémy, Jouve, \& Lelord, 1986; Hegerl \& Juckel, 1993). It would be interesting to investigate whether the augmenting tendency in children is associated with a less efficient functioning of these neurotransmitter systems.

\section{REFERENCES}

Allison, T., Hume, A. L., Wood, C. C., \& Goff, W. R. (1984). Developmental and aging changes in somatosensory, auditory and visual evoked potentials. Electroencephalography and Clinical Neurophysiology, 58, 14-24.

American Electroencephalographic Society. (1991). American Electroencephalographic Society guidelines for standard electrode position nomenclature. Journal of Clinical Neurophysiology, 8, 200-202.

Barratt, E. S., Pritchard, W. S., Faulk, D. M., \& Brandt, M. E. (1987). The relationship between impulsiveness subtraits, trait anxiety and visual N100 augmenting0reducing: A topographic analysis. Personality and Individual Differences, 8, 43-51.

Blenner, J. L., \& Yingling, C. D. (1993). Modality specificity of evoked potential augmentingOreducing. Electroencephalography and Clinical Neurophysiology, 88, 131-142.

Bruneau, N., Barthélémy, C., Jouve, J., \& Lelord, G. (1986). Frontal auditory-evoked potential augmenting-reducing and urinary homovanillic acid. Neuropsychobiology, 16, 78-84.

Bruneau, N., Barthélémy, C., Roux, S., Jouve, J., \& Lelord, G. (1989). Auditory evoked potential modifications according to clinical and biochemical responsiveness to fenfluramine treatment in children with autistic behavior. Neuropsychobiology, $21,48-52$.

Bruneau, N., Roux, S., Garreau, B., \& Lelord, G. (1985). Frontal auditory evoked potentials and augmenting0reducing. Electroencephalography and Clinical Neurophysiology, 62, 364-371.

Bruneau, N., Roux, S., Guérin, P., Barthélémy, C., \& Lelord, G. (1997). Temporal prominence of auditory evoked potentials (N1 wave) in 4-8-year-old children. Psychophysiology, 34, 32-38.

Buchsbaum, M. S., Haier, R. J., \& Johnson, J. (1983). Individual differences in augmenting0reducing evoked potentials. In A. Gale \& J. A. Edwards (Eds.), Physiological correlates of human behavior: Vol. 3.

Individual differences and psychopathology (pp. 117-138). New York: Academic Press.

Buchsbaum, M. S., \& Pfefferbaum, A. (1971). Individual differences in stimulus intensity response. Psychophysiology, 8, 600- 611.

Buchsbaum, M. S., \& Silverman, J. (1968). Stimulus intensity and the cortical evoked response. Psychosomatic Medicine, XXX(1), 12-22.

Carrillo-de-la-Peña, M. T. (1992). ERP augmentingOreducing and sensation-seeking: a critical review. International Journal of Psychophysiology, 12, 211-220. 
Como, P. G., Simons, R., \& Zuckerman, M. (1984). Psychophysiological indices of sensation seeking as a function of stimulus intensity. Psychophysiology, 21, 572-572.

Connolly, J. F., \& Gruzelier, J. H. (1982). Amplitude and latency changes in the visual evoked potential to different stimulus intensities. Psychophysiology, 19, 599- 608.

Courchesne, E. (1978). Neurophysiological correlates of cognitive development: Changes in long-latency event-related potentials from childhood to adulthood. Electroencephalography and Clinical Neurophysiology, 45, 468- 482.

Dustman, R. E., Emmerson, R. Y., \& Shearer, D. E. (1996). Life span changes in electrophysiological measures of inhibition. Brain and Cognition, 30, 109-126.

Dustman, R. E., Shearer, D. E., \& Emmerson, R. Y. (1993). EEG and event-related potentials in normal aging. Progress in Neurobiology, 41, 369-401.

Dustman, R. E.; Shearer, D. E., \& Snyder, E. W. (1982). Age differences in augmentingOreducing of occipital visually evoked potentials. Electroencephalography and Clinical Neurophysiology, 54, 99-110.

Dustman, R. E., \& Snyder, E. W. (1981). Life-span changes in visually evoked potentials at central scalp. Neurobiology of Aging, 2 , 303-308.

Dustman, R. E., Snyder, E. W., \& Schlehuber, C. J. (1981). Life-span alterations in visually evoked potentials and inhibitory function. Neurobiology of Aging, 2, 187-192.

Dykman, R.A., Holcomb, P. J., Ackerman, P. T., \& McCray, D. S. (1983). Auditory ERP augmentation-reduction and methylphenidate dosage needs in attention and reading disordered children. Psychiatry Research, 9, 255-269.

Hegerl, U., Gallinat, J., \& Mrowinski, D. (1994). Intensity dependence of auditory evoked dipole source activity. International Journal of Psychophysiology, 17, 1-3.

Hegerl, U., \& Juckel, G. (1993). Intensity dependence of auditory evoked potentials as an indicator of central serotonergic neurotransmission—Arguments for a new hypothesis. Biological Psychiatry, 33, 173-187. Kaskey, G. B., Salzman, L. F., Klorman, R., \& Pass, H. L. (1980). Relationships between stimulus intensity and amplitude of visual and au-

ditory event related potentials. Biological Psychology, 10, 115-125.

Johnson, R. (1989). Development evidence for modality-dependent P300 generators: A normative study. Psychophysiology, 26, 651- 667 .

Lolas, F., Collin, C., Camposano, S., Etcheberrigaray, R., \& Rees, R.

(1987). Hemispheric asymmetry of augmenting0reducing in visual and auditory evoked potentials. Biological Psychiatry, 22, 14131416.

Martín, L., Barajas, J.J., Fernandez, R., \& Torres, E. (1988). Auditory event-related potentials in well-characterized groups of children. Electroencephalography and Clinical Neurophysiology, 71, 375-381.

Näätänen, R., \& Picton, T. (1987). The N1 wave of the human electric and magnetic response to sound: A review and an analysis of the component structure. Psychophysiology, 24, 375- 425.

Orlebeke, J. F., Kok, A., \& Zeillemaker, C. W. (1984) Augmenting-

reducing (disinhibition) and the processing of auditory stimulus intensity: An ERP study. Psychophysiology, $21,591$.

Petrie, A. (1960). Some psychological aspects of pain and the relief of suffering. Annals of the New York Academy of Sciences, 86, 13-27.

Petrie, A. (1978). Individuality in pain and suffering (2nd ed.). Chicago: University of Chicago Press.

Pineda, J. A., Holmes, T. C., \& Foote, S. L. (1991). Intensity-amplitude relationships in monkey event-related potentials: Parallels to human augmenting-reducing responses. Electroencephalography and Clinical Neurophysiology, 78, 456- 465.

Prescott. J., Connolly, J. F., \& Gruzelier, J. H. (1984). The augmenting0 reducing phenomenon in the auditory evoked potential. Biological Psychology, 19, 31- 44.

Raine, A., Mitchell, D. A., \& Venables, P. H. (1981). Cortical augmenting-reducing: Modality specific? Psychophysiology, 18, 700708.

Saxton, P. M., Siegel, J., \& Lukas, J. H. (1987). Visual evoked potential augmentingOreducing slopes in cats—I. Reliability as a function of flash intensity range. Personality and Individual Differences, 8, 499- 509.

Vasey, M. W., \& Thayer, J. F. (1987). The continuing problem of false positives in repeated measures ANOVA in psychophysiology: A multivariate solution. Psychophysiology, 24, 479- 486. 